\title{
Dynamic Stiffness Analysis and Experimental Verification of Axial Magnetic Bearing Based on Air Gap Flux Variation in Magnetically Suspended Molecular Pump
}

\author{
Jinji Sun ${ }^{1,2}$, Wanting Wei ${ }^{1 *}$, Jiqiang Tang ${ }^{1}$ and Chun-E Wang ${ }^{3}$
}

\begin{abstract}
Current and displacement stiffness are important parameters of axial magnetic bearing (AMB) and are usually considered as constants for the control system. However, in actual dynamic work situations, time-varying force leads to time-varying currents and air gap with a specific frequency, which makes the stiffness of appear decrease and even worsens control performance for the whole system. In this paper, an AMB dynamic stiffness model considering the flux variation across the air gap due to frequency is established to obtain the accurate dynamic stiffness. The dynamic stiffness characteristics are analyzed by means of the dynamic equivalent magnetic circuit method. The analytical results show that the amplitude of current and displacement stiffness decreases with frequency increasing. Moreover, compared with the stiffness model without considering the variation of flux density across the air gap, the improved dynamic stiffness results are closer to the actual results. Through the dynamic stiffness measurement method of AMB, experiments of AMB in magnetically suspended molecular pump (MSMP) are carried out and the experimental results are consistent with theoretical analysis results. This paper proposes the dynamic stiffness model of axial magnetic bearing considering the variation of flux density across the air gap, which improves the accuracy of the AMB stiffness analysis.
\end{abstract}

Keywords: Axial magnetic bearing (AMB), Dynamic stiffness, Magnetically suspended molecular pump (MSMP)

\section{Introduction}

Molecular pump is a kind of high-end scientific instrument for obtaining high vacuum environment, such as cyclotron, laser, mass spectrometer, gyro equipment, and so on [1-3]. The magnetic bearing has broad prospects in industrial applications [4-6] compared with the traditional bearing because of its special advantages, such as non-contact, no friction, low power consumption, low maintenance cost, dynamical controllability and active

\footnotetext{
*Correspondence: weiwanting@buaa.edu.cn

1 Key Laboratory of Fundamental Science for National Defense,

Novel Inertial Instrument and Navigation System Technology, School

of Instrument Science and Opto-Electronics Engineering, Beihang

University, Beijing 100191, China

Full list of author information is available at the end of the article
}

control ability of rotor dynamic imbalance. The magnetic bearing used as the support unite in magnetically suspended molecular pump (MSMP) can realize the oil-free and wear-free operation of the molecular pump, quiet operation and the minimal vibration, which is especially suitable for the semiconductor industry, such as ultrahigh vacuum applications [7-9].

In most magnetically suspended molecular pumps, the support unite of magnetic bearing includes radial active magnetic bearing (RAMB) and axial magnetic bearing (AMB). The magnetic bearings as the supporting parts are used to maintained the stable suspension. In the actual situation, the magnetic bearings are always subject to time-varying forces. The coil currents are changed to adjust the bearing force in response to the applied load. 
These time-varying currents and air gap will cause flux variations in the magnetic path, and hence inducing eddy currents, which not only result in a power loss of the system but also cause magnitude decrease of the bearing force and stiffness. This will affect the dynamic performance and stability of the whole system [10-12].

Several studies have been carried out to develop analytical models for magnetic bearings from different aspects. Kucera et al. [13] presented an analytical method of axial bearings considering eddy currents, and the magnetic field solutions for a semi-infinite plate were used to approximate the flux distributions. The analytical solution for the flux, the impedance and the force of an axial bearing can be derived. The traditional magnetic circuit method is improved by considering the dynamic effective reluctance depended on frequency [14-16]. The cylindrical magnetic actuator (The cylindrical magnetic actuator has the same working principle as the axial magnetic bearing.) was divided into several elements and the frequency-dependent reluctance of each element was obtained according to the flux distribution, and then derived the dynamic force and stiffness of the axial magnetic bearing. Based on these studies, according to the actual situations, Sun et al. [17] were extended to include the magnetic actuator with a center hole common in rotating machinery, and factors such as saturation, leakage, and fringing flux were considered. A simple magnetic circuit model including eddy currents was presented, from which the stiffness can be derived analytically. A magnetic circuit calculation model is proposed for the core eddy current loss caused by the axial high frequency vibration of axial magnetic bearing $[18,19]$. The dynamic air gap flux and electromagnetic force are analyzed. By analytical and finite element method, the segmented AMBs were developed to reducing the effect of eddy currents $[20,21]$. The results demonstrate that the segmentation of the stator results in dramatic improvements in actuator dynamic performance. Considering the driving method of excitation, an analytical model for a solid-core AMB including the eddy-current effect under voltage drive based on the magnetic circuit method was presented in Ref. [22], which indicates the voltage drive can effectively reduce the dynamic performance.

Moreover, the measurement of dynamic stiffness is especially important. Ref. [23] proposed the axial displacement stiffness measurement method of the axial passive magnetic bearing and verified the correctness in the magnetically suspended control momentum gyro. In Ref. [24], a new stiffness measurement method of repulsive passive axial magnetic bearing with $\mathrm{Hal}-$ bach magnetic array is put forward. Ref. [25] proposed a new stiffness measurement method for magnetically suspended flywheel to measure current stiffness and displacement stiffness of permanent magnet biased radial magnetic bearing. Refs. [26, 27] described a detailed stiffness measurement method for a radial hybrid magnetic bearing. The above researches for stiffness measurement consider the stiffness of magnetic bearing as a constant. However, some researchers have found that the stiffness of magnetic bearing would decrease significantly with the field frequency increasing, which would influence the stability of radial magnetic bearing system [28, 29]. Sun et al. [17] carried out the experiment about the dynamic stiffness including eddy currents, but the specific experimental methods are not described.

In conclusion, early models of solid magnetic actuators assumed that the flux density in the air gap was homogeneous because no eddy current is produced in the air gap. However, the flux density varies with axial position along the air gap. Neglecting the flux variation across the air gap during the analytical stage will bring the inaccurate stiffness result. The inaccurate analytical model result in the biased feedback for control system and even worsen control performance for the whole system. This variation of flux density across the air gap is an important aspect of actuator behavior that must be captured in high fidelity models. Furthermore, in order to verify the correctness of analytical dynamic stiffness model, it is necessary to measure the actual dynamic stiffness.

In this paper, considering the dynamic characteristics that time-varying force leads to time-varying currents and air gap with a specific frequency, the dynamic stiffness model of axial magnetic bearing including the eddy-current effect considering the variation of flux density across the air gap is firstly built up by analyzing the dynamic equivalent magnetic circuit model. Then, the dynamic stiffness measurement method is adopted in Magnetically Suspended Molecular Pump to verify the validity of the theoretical analyze results.

\section{Magnetic Circuit Model of Axial Magnetic Bearing for MSMP}

The magnetically suspended molecular pump model is shown Figure 1, which is mainly composed of one rotor shaft, two RAMBs, one axial active magnetic bearing, one high-speed BLDCM, two integrated displacement sensors and stator blades. The configuration of the AMB is pictured in Figure 2, the materials of the stator and rotor core are silicon steels and the corresponding parameters are described in Table 1.

The traditional static magnetic circuit theory has been widely used in the design of an AMB. The magnetic circuit model consists of the magneto motive force (MMF) determined by coil turns and currents and the reluctance of inner and outer air gap. The flux and bearing force can be derived conveniently with the reluctance of the air gap 


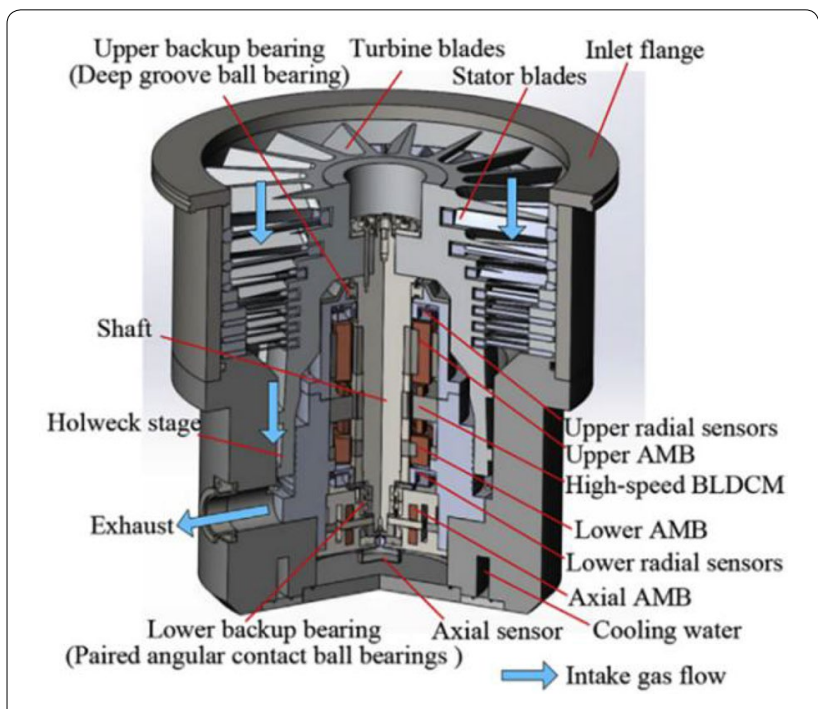

Figure 1 Magnetically suspended molecular pump model

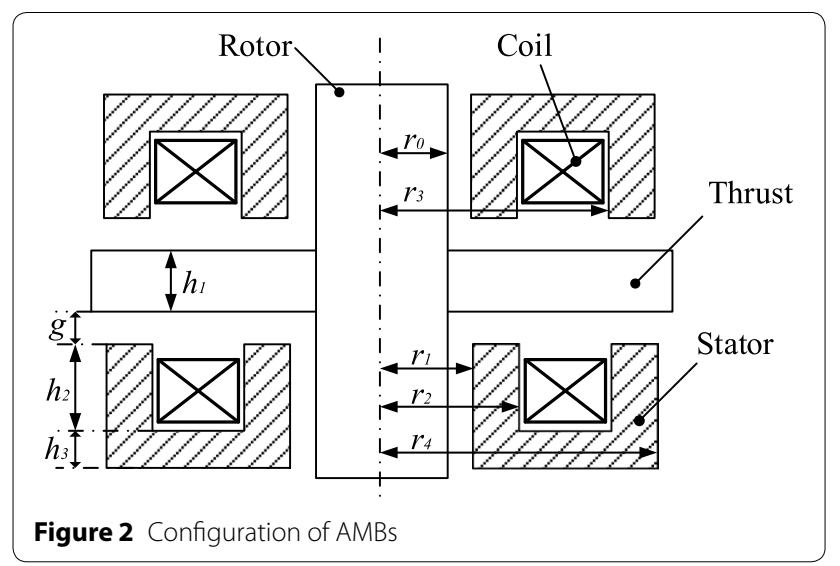

and MMF applied. However when the load force of the magnetic bearing varies with a specific frequency, the time-varying force leads to time-varying currents and air gap, which generates eddy currents in the rotor and stator. This situation referred to in this paper that the timevarying force leads to time-varying currents and air gap is defined as dynamic characteristics. In this case, the reluctance of the iron core varies influenced by eddy current effect. According to the analysis method of effective magnetic reluctance in Ref. [10], the eddy current formed by the alternating magnetic field on the solid iron core is equivalent in the form of eddy current magnetic reluctance, and then the air gap flux and electromagnetic force can be calculated according to the equivalent magnetic circuit method.

In order to obtain the effective magnetic reluctance on the iron core, the magnetic path of the axial
Table 1 Main parameters of AMB

\begin{tabular}{|c|c|c|}
\hline Description & Parameter & Value \\
\hline Rotor outer diameter & $r_{0}(\mathrm{~mm})$ & 8 \\
\hline $\begin{array}{l}\text { Inner magnetic conducting ring outer } \\
\text { diameter }\end{array}$ & $r_{1}(\mathrm{~mm})$ & 14 \\
\hline $\begin{array}{l}\text { Inner magnetic conducting ring inner } \\
\text { diameter }\end{array}$ & $r_{2}(\mathrm{~mm})$ & 17 \\
\hline $\begin{array}{l}\text { Outer magnetic conducting ring inner } \\
\text { diameter }\end{array}$ & $r_{3}(\mathrm{~mm})$ & 27 \\
\hline $\begin{array}{l}\text { Outer magnetic conducting ring outer } \\
\text { diameter }\end{array}$ & $r_{4}(\mathrm{~mm})$ & 30 \\
\hline Air gap length & $g(\mathrm{~mm})$ & 0.35 \\
\hline Thrust plate thickness & $h_{1}(\mathrm{~mm})$ & 4 \\
\hline Axial length of winding slot & $h_{2}(\mathrm{~mm})$ & 6.4 \\
\hline $\begin{array}{l}\text { Axial length of outside magnetic conduct- } \\
\text { ing ring }\end{array}$ & $h_{3}(\mathrm{~mm})$ & 8.5 \\
\hline Number of turns & $N$ (turns/pole) & 300 \\
\hline Bias current & $I_{0}(\mathrm{~A})$ & 0.5 \\
\hline Relative permeability & $\mu$ & 1000 \\
\hline Conductivity & $\sigma(\mathrm{S} / \mathrm{m})$ & $7.46 \times 10^{6}$ \\
\hline
\end{tabular}

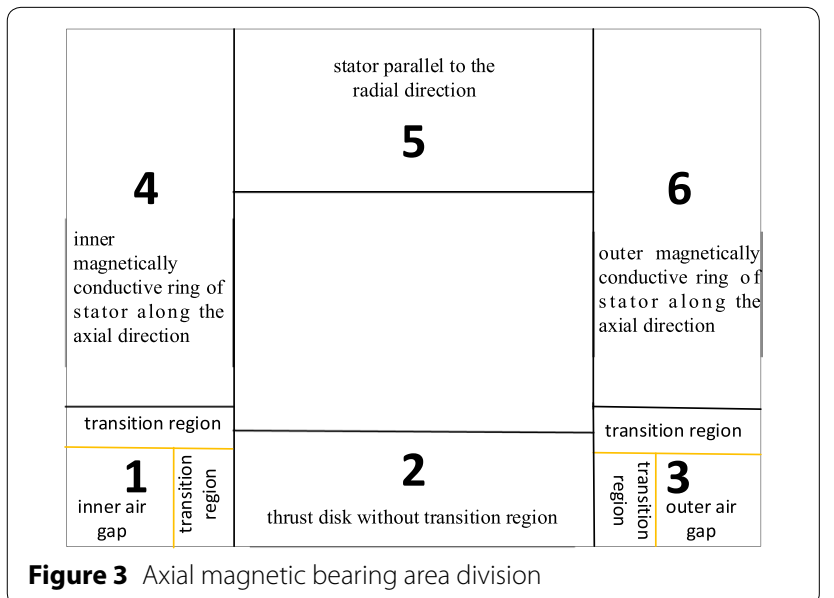

magnetic bearing is divided into several different parts (ignoring magnetic leakage), as shown in Figure 3. The region 1 includes a transition region at the top of the inner magnetic pole, an air gap corresponding to the inner magnetic pole, and a transition region in the thrust disk corresponding to the inner magnetic pole. The region 3 includes a transition region at the top of the outer magnetic pole, an air gap corresponding to the outer magnetic pole, and a transition region in the corresponding thrust disk. In region 1 and 3, the magnetic lines of force in the transition region of the thrust disk are distributed in the radial direction, and the magnetic lines of force in the air gap are parallel to the axial direction. The region 2 is other than the region 
1 and the region 3 in the thrust disk part. The region 5 is the portion corresponding to the region 2 in the stator, the magnetic lines of force in the region 2 and the region 5 are all parallel to the radial direction. The region 4 is the portion of the inner magnetically conductive ring from which the region 1 is removed, and the region 6 is the outer portion.

The corresponding equivalent reluctance is calculated in each part of the core, and the equivalent magnetoresistance expression is simplified by Ad Hoc approximation and Taylor series and pade approximation respectively. Finally, the equivalent reluctance of each region can be obtained. According to Ref. [10], the effective reluctances expression of the elements is:

$$
\begin{aligned}
& R_{1}^{0}=\frac{g}{\pi \mu_{0}\left(r_{2}^{2}-r_{1}^{2}\right)}, \\
& R_{2}=\frac{\ln \left(r_{3} / r_{2}\right)}{2 \pi h_{1} \mu_{r} \mu_{0}}+\frac{\ln \left(r_{3} / r_{2}\right)}{2 \pi} \sqrt{\frac{\sigma}{\mu_{r} \mu_{0}}} \sqrt{s}, \\
& R_{3}^{0}=\frac{g}{\pi \mu_{0}\left(r_{4}^{2}-r_{3}^{2}\right)}, \\
& R_{4}=\frac{h_{2}}{\pi \mu_{r} \mu_{0}\left(r_{2}^{2}-r_{1}^{2}\right)}+\frac{h_{2}}{2 \pi r_{2}} \sqrt{\frac{\sigma}{\mu_{r} \mu_{0}}} \sqrt{s}, \\
& R_{5}=\frac{\ln \left(r_{3} / r_{2}\right)}{2 \pi h_{3} \mu_{r} \mu_{0}}+\frac{\ln \left(r_{3} / r_{2}\right)}{2 \pi} \sqrt{\frac{\sigma}{\mu_{r} \mu_{0}}} \sqrt{s}, \\
& R_{6}=\frac{h_{2}}{\pi \mu_{r} \mu_{0}\left(r_{4}^{2}-r_{3}^{2}\right)}+\frac{h_{2}}{2 \pi r_{3}} \sqrt{\frac{\sigma}{\mu_{r} \mu_{0}}} \sqrt{s} .
\end{aligned}
$$

Each dynamic equivalent reluctance can be expressed as:

$$
R_{j}=R_{j}^{0}+R_{j}^{e}=R_{j}^{0}+c_{j} \sqrt{s}, \quad j=1, \ldots, 6 .
$$

$R_{j}^{0}$ is the static reluctance without eddy current, $R_{j}^{e}=c_{j} \sqrt{s}$ is the dynamic reluctance including eddy current.

Although there is no eddy current is in the air gap, due to eddy-current effect in the iron core, the flux density in solid actuators varies across the air gap. According to Ref. [9], if there is no flux leak, the magnetic flux of magnetic bearing is:

$$
\phi=\frac{\mu_{0} N A I}{2 x+\frac{l_{f_{e}}}{\mu_{r}} \frac{\gamma}{\tanh (\gamma)}}=\mu_{0} N A I \frac{1}{2 g} \frac{1}{1+\frac{1}{a_{f e} \frac{\gamma}{\tanh (\gamma)}},}
$$

$$
\begin{aligned}
& a_{f e}=\mu_{r} \frac{2 g}{l_{f e}}, \\
& \gamma=h_{1} \sqrt{s \mu \sigma},
\end{aligned}
$$

where $l_{f e}$ is the length of the solid core. Dynamic reluctance of inner and outer air gap including eddy current is:

$$
R_{(1,3)}^{e}=\frac{N I}{\phi}=\frac{2 g\left[1+\frac{1}{a_{f e}} \frac{\gamma}{\tanh (\gamma)}\right]}{\mu_{0} A_{(n, w)}},
$$

where $A_{n}$ and $A_{\mathrm{w}}$ is the section surface of inner and outer air gap respectively.

Considering the flux density variation across the air gap, the equivalent magnetic circuit diagram of this axial electromagnetic magnetic bearing is shown in Figure 4.

Where the internal air gap magnetic fluxes $\phi_{n}$ and external air gap magnetic fluxes $\phi_{w}$ are equal. The dynamic total reluctance based on the flux variation can be expressed as:

$$
R_{t}=\sum_{j=1}^{6} R_{j}=\sum_{j=1}^{6} R_{j}^{0}+\sum_{j=1}^{6} R_{j}^{e} .
$$

The static total reluctance can be expressed as:

$$
R_{t}^{0}=\sum_{j=1}^{6} R_{j}^{0}
$$

\section{Analysis of Dynamic Stiffness of AMB FOR MSMP}

According to the above analysis, considering the influence of the eddy-current effect including the flux density variation across the air gap, it can be seen from Figure 4 that when the thrust disk is stably suspended in the center position of the magnetic bearing, the internal and external air gap magnetic fluxes are equal, where $\phi_{w}$ is the magnetic flux at the outer magnetic pole air gap and $\phi_{n}$ is the magnetic flux at the inner magnetic pole air gap.

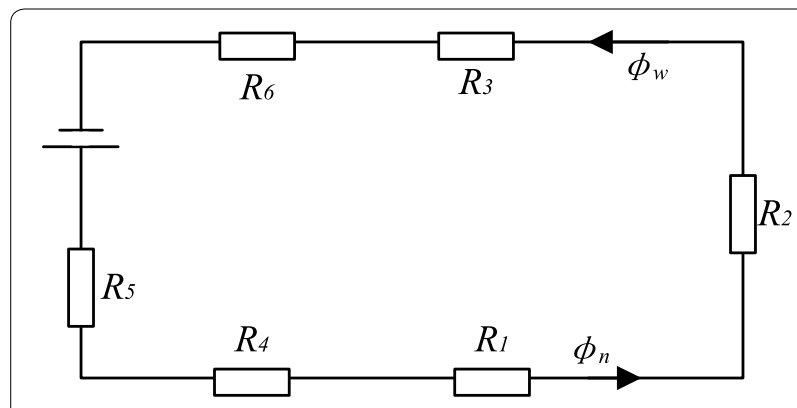

Figure 4 Dynamic equivalent magnetic circuit model of AMB 


$$
\phi_{n}=\phi_{w}=\frac{N I_{0}}{R_{t}} .
$$

The force of the single-sided magnetic bearing (one side of the thrust plate) is:

$$
\begin{gathered}
F=\frac{\phi_{n}^{2}}{2 \mu_{0} A_{n}}+\frac{\phi_{w}^{2}}{2 \mu_{0} A_{w}}=N^{2} I_{0}^{2}\left(\frac{1}{A_{n}}+\frac{1}{A_{w}}\right) / \\
2 \mu_{0}\left[R_{t}^{0}+\left(\frac{\ln \left(r_{3} / r_{2}\right)}{\pi}+\frac{h_{2}}{2 \pi r_{2}}+\frac{h_{2}}{2 \pi r_{3}}\right) \sqrt{\frac{\sigma}{\mu_{r} \mu_{0}}} \sqrt{j w}\right. \\
\left.+\frac{2 g\left[1+\frac{1}{a_{f e}} \frac{h_{1} \sqrt{j w \mu \sigma}}{\tanh \left(h_{1} \sqrt{j w \mu \sigma}\right)}\right]}{\mu_{0}}\left(\frac{1}{A_{n}}+\frac{1}{A_{w}}\right)\right]^{2} .
\end{gathered}
$$

The relationship between dynamic force and frequency is shown in Figure 5, which illustrates that as the frequency increases, the dynamic force appears amplitude attenuation.

\subsection{Dynamic Current Stiffness}

The bias current $I_{0}$ and the differential alternating control current $i_{c}=i e^{j \omega t}$ are applied to the above and below coils of the thrust disk, namely,

$$
I=I_{0} \pm i e^{j \omega t} .
$$

The magnetic fluxes on the above and below sides of the thrust plate are:

$$
\left\{\begin{array}{l}
\phi_{a}=\phi_{0}+\phi_{i}, \\
\phi_{b}=\phi_{0}-\phi_{i}
\end{array}\right.
$$

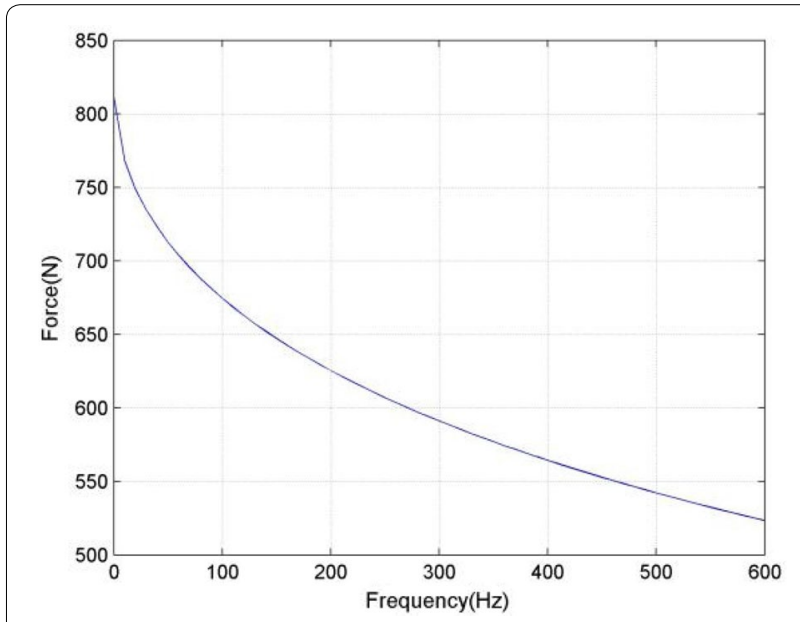

Figure 5 Dynamic axial force value at different frequency where $\phi_{0}$ is the static bias magnetic flux generated by the bias current, $\phi_{i}$ is the dynamic magnetic flux generated by the coils considering the eddy-current effect including the flux density variation across the air gap, expressed as:

$$
\begin{aligned}
\phi_{0}= & \frac{N I_{0}}{R_{t}^{0}}=\pi N I_{0} /\left[\frac{g}{\mu_{0}\left(r_{2}^{2}-r_{1}^{2}\right)}+\frac{\ln \left(r_{3} / r_{2}\right)}{2 h_{1} \mu_{r} \mu_{0}}+\frac{g}{\mu_{0}\left(r_{4}^{2}-r_{3}^{2}\right)}\right. \\
& \left.+\frac{h_{2}}{\mu_{r} \mu_{0}\left(r_{2}^{2}-r_{1}^{2}\right)}+\frac{\ln \left(r_{3} / r_{2}\right)}{2 h_{3} \mu_{r} \mu_{0}}+\frac{h_{2}}{\mu_{r} \mu_{0}\left(r_{4}^{2}-r_{3}^{2}\right)}\right], \\
\phi_{i}= & \frac{N i e^{j w t}}{R_{t}}=N i e^{j w t} /\left[R_{t}^{0}+\left(\frac{\ln \left(r_{3} / r_{2}\right)}{\pi}+\frac{h_{2}}{2 \pi r_{2}}+\frac{h_{2}}{2 \pi r_{3}}\right)\right. \\
& \left.\times \sqrt{\frac{\sigma}{\mu_{r} \mu_{0}}} \sqrt{j w}+\frac{2 g\left[1+\frac{1}{a_{f_{e}}} \frac{h_{1} \sqrt{j w \mu \sigma}}{\tanh \left(h_{1} \sqrt{j w \mu \sigma}\right)}\right.}{\mu_{0}}\left(\frac{1}{A_{n}}+\frac{1}{A_{w}}\right)\right] .
\end{aligned}
$$

The resultant force of the above and below sides of the magnetic bearing can be obtained as:

$$
\begin{aligned}
F= & \frac{\left(\phi_{0}+\phi_{i}\right)^{2}}{2 \mu_{0} A_{n}}+\frac{\left(\phi_{0}+\phi_{i}\right)^{2}}{2 \mu_{0} A_{w}}-\frac{\left(\phi_{0}-\phi_{i}\right)^{2}}{2 \mu_{0} A_{n}} \\
& -\frac{\left(\phi_{0}-\phi_{i}\right)^{2}}{2 \mu_{0} A_{w}}=\frac{2 \phi_{0} \phi_{i}}{\mu_{0} A_{n}}+\frac{2 \phi_{0} \phi_{i}}{\mu_{0} A_{w}} .
\end{aligned}
$$

Current stiffness is calculated as:

$$
\begin{gathered}
k_{i}(\omega)=\left.\frac{\partial F}{\partial i}\right|_{i_{c}=0}=\frac{2 N^{2} I_{0}}{\mu_{0} R_{t}^{o} R_{t}}\left(\frac{1}{A_{n}}+\frac{1}{A_{w}}\right)=2 N^{2} I_{0}\left(\frac{1}{A_{n}}+\frac{1}{A_{w}}\right) / \\
{\left[\mu _ { 0 } R _ { t } ^ { o } \left(R_{t}^{0}+\left(\frac{\ln \left(r_{3} / r_{2}\right)}{\pi}+\frac{h_{2}}{2 \pi r_{2}}+\frac{h_{2}}{2 \pi r_{3}}\right) \sqrt{\frac{\sigma}{\mu_{r} \mu_{0}}} \sqrt{j w}\right.\right.} \\
\left.+\frac{2 g\left[1+\frac{1}{a_{f e}} \frac{h_{1} \sqrt{j w \mu \sigma}}{\tanh \left(h_{1} \sqrt{j w \mu \sigma}\right)}\right]}{\mu_{0}}\left(\frac{1}{A_{n}}+\frac{1}{A_{w}}\right)\right]
\end{gathered}
$$

The relationship between dynamic current displacement stiffness and frequency is shown in Figure 6, which illustrates that as the frequency increases, the current stiffness appears amplitude attenuation.

\subsection{Dynamic Displacement Stiffness}

Keep the bias current in the coil unchanged, assuming that the rotor makes a small harmonic vibration at the center position expressed as $g_{z} e^{j \omega t}$, the above and below axial air gap can be expressed as:

$$
z=g \pm g_{z} e^{j \omega t} .
$$

In this case, the air gap reluctance of above and below magnetic poles is expressed as: 


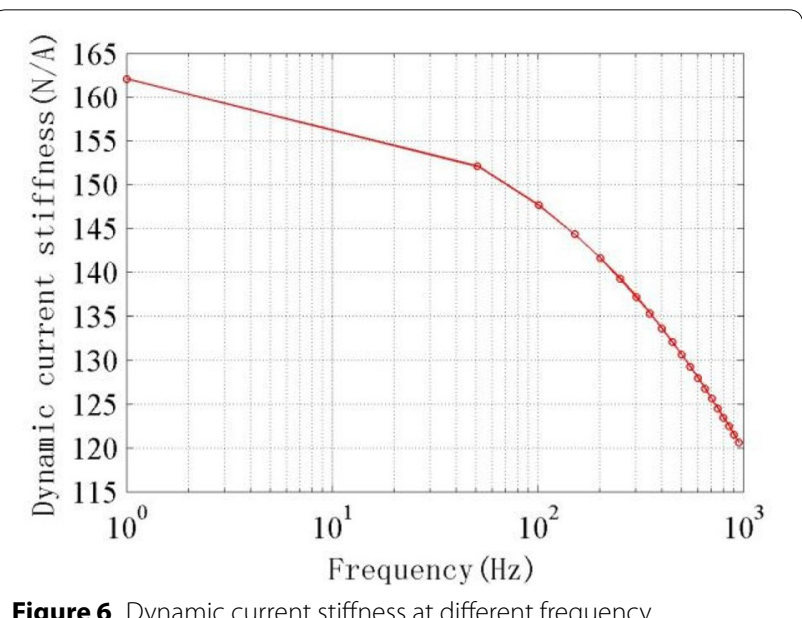

Figure 6 Dynamic current stiffness at different frequency

$$
\begin{aligned}
& R_{a g}=R_{g 0}+R_{g z} e^{j \omega t}, \\
& R_{b g}=R_{g 0}-R_{g z} e^{j \omega t},
\end{aligned}
$$

where $R_{g 0}$ is the static air gap reluctance, expressed as $R_{g 0}=\frac{g}{\mu_{0}}\left(\frac{1}{A_{n}}+\frac{1}{A_{w}}\right) ; R_{g z}$ is the dynamic air gap reluctance generated by the magnetic pole vibration, expressed as $R_{g z}=\frac{g_{z}}{\mu_{0}}\left(\frac{1}{A_{n}}+\frac{1}{A_{w}}\right)$; Ignoring the magnetic flux leakage, then the magnetic flux of the above and below air gaps can be expressed as:

$$
\begin{aligned}
\phi_{a} & =\frac{N I_{0}}{R_{t}+R_{g z} e^{j w t}}, \\
\phi_{b} & =\frac{N I_{0}}{R_{t}-R_{g z} e^{j w t}} .
\end{aligned}
$$

Since $g_{z}$ is much smaller than $g, R_{g z}$ is much smaller than $R_{t}$, then Eq. (25) can perform Taylor expansion, leaving only the first two terms, then the following formula can be obtained:

$$
\begin{aligned}
& \phi_{a} \approx \phi_{0}+\phi_{n z}, \\
& \phi_{b} \approx \phi_{0}-\phi_{n z}, \\
& \phi_{0}=\frac{N I_{0}}{R_{t}^{0}} \\
& \phi_{n z}=-\frac{N I_{0} R_{g z}}{R_{t}^{0} R_{t}} e^{j w t} .
\end{aligned}
$$

In the same way as current stiffness, the formula for calculating the displacement stiffness considering the eddy-current effect including the flux density variation

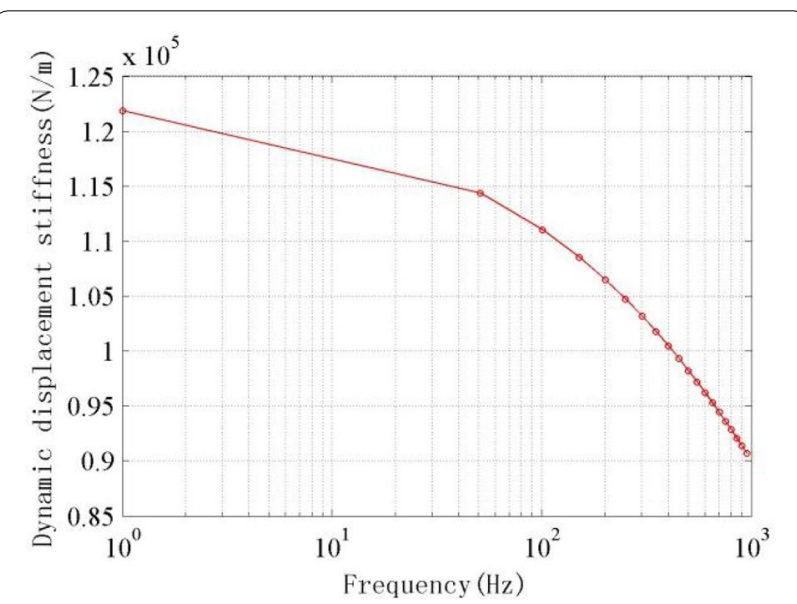

Figure 7 Dynamic displacement stiffness at different frequency

across the air gap (the resultant force on both sides of the thrust disk) is:

$$
\begin{aligned}
k_{z}(\omega)= & \left.\frac{\partial F_{z}}{\partial z}\right|_{g_{z}=0}=-\frac{2 N^{2} I_{0}^{2}}{\mu_{0}^{2}\left(R_{t}^{0}\right)^{2} R_{t}}\left(\frac{1}{A_{n}^{2}}+\frac{1}{A_{w}^{2}}\right) \\
= & -2 N^{2} I_{0}^{2}\left(\frac{1}{A_{n}^{2}}+\frac{1}{A_{w}^{2}}\right) /\left[\mu _ { 0 } ^ { 2 } ( R _ { t } ^ { 0 } ) ^ { 2 } \left(R_{t}^{0}\right.\right. \\
& +\left(\frac{\ln \left(r_{3} / r_{2}\right)}{\pi}+\frac{h_{2}}{2 \pi r_{2}}+\frac{h_{2}}{2 \pi r_{3}}\right) \sqrt{\frac{\sigma}{\mu_{r} \mu_{0}}} \sqrt{j w} \\
& \left.+\frac{2 g\left[1+\frac{1}{a_{f e}} \frac{h_{1} \sqrt{j w \mu \sigma}}{\tanh \left(h_{1} \sqrt{j w \mu \sigma}\right)}\right]}{\mu_{0}}\left(\frac{1}{A_{n}^{2}}+\frac{1}{A_{w}^{2}}\right)\right],
\end{aligned}
$$

where $N$ represents the number of turns, $I_{0}$ is the bias current.

The relationship between dynamic displacement stiffness and frequency is shown in Figure 7, which illustrates that as the frequency increases, the displacement stiffness appears amplitude attenuation.

\section{Dynamic Stiffness Measurement Method}

The stiffness obtained by analytical model may not satisfy the requirements of actual magnetic bearing design and application, so it is important to measure the actual stiffness value of the magnetic bearing. This chapter adopts a magnetic bearing stiffness test method, which can measure the current stiffness and displacement stiffness of the magnetic bearing rotor at a certain speed and be used in the molecular pump system to test the axial magnetic bearing dynamic stiffness test. The step of this method 
is first to adjust the reference value of the displacement sensor, then stabilize the rotor in a certain position by the magnetic bearing itself and inject a sinusoidal signal into the currents of the bearing to excite the closed-loop system, finally measure the control current of the corresponding position magnetic bearing and calculate the stiffness of the magnetic bearing.

When the magnetic bearing rotor system is stably suspended, a sinusoidal signal was injected into the currents of the bearing to represent the time-varying currents and air gap with a specific frequency. The electromagnetic force of the magnetic bearing at this time is a dynamic force related to frequency. In this paper, gravity is considered as the bearing force of the magnetic bearing. Due to the molecular pump structure of the internal rotor, it is difficult to implement the force sensor, and the actual means of measuring force are limited. In addition, the dynamic force affected by frequency is much smaller than gravity. Therefore, we use gravity to equivalent dynamic electromagnetic force. The measured channel should be adjusted to the same direction of gravity during the test. Because the mechanical structure and mass distribution of the whole system are the known conditions, the electromagnetic force of the measured magnetic bearing channel can be expressed as a function of gravity by force analysis.

$$
F \approx f(m g),
$$

where $F$ is the electromagnetic force of the magnetic bearing measured channel, $m$ is the mass of the rotor of magnetic bearing system.

Because the electromagnetic force can be linearized near the center of the magnetic bearing, which can be expressed:

$$
i(\omega)=-\frac{k_{h}(\omega)}{k_{i}(\omega)}\left(h-h_{0}\right)+\frac{f(m g)}{k_{i}(\omega)},
$$

where $k_{i}(\omega)$ and $k_{h}(\omega)$ are the current stiffness and displacement stiffness, $i(\omega)$ is the control current, $h$ is the rotor displacement, and $h_{0}$ is the magnetic center position of the magnetic bearing to be tested.

When the rotor system runs at a specific speed, the rotor is adjusted to a position $h_{1}$ near the magnetic center, and the corresponding control current can be measured as $i_{1}(\omega)$, then the current stiffness and displacement stiffness can be calculated by Eqs. (32) and (33). In the magnetic bearing control system, the displacement of rotor is directly measured by eddy current displacement sensor, compared with the reference signal of displacement set in the program of magnetic bearing control system, and then the PID operation is performed to control the rotor to suspend stably at the reference position. The reference signal can be adjusted online by the control program. Therefore, at a certain frequency, the change of rotor position can be achieved by adjusting the displacement reference signal online. The reference signal of displacement 2048 is used as the central position to adjust the rotor stable suspension position. The reference signal of displacement adjustment range is $1600-2500$, and the corresponding rotor suspension position is $-0.04 \sim 0.04$ $\mathrm{mm}$.

$$
\begin{aligned}
& k_{i}(\omega)=\frac{f(m g)}{i_{0}(\omega)}, \\
& k_{h}(\omega)=\frac{f(m g)-k_{i}(\omega) \cdot\left(i_{1}(\omega)-i_{0}(\omega)\right)}{h_{1}-h_{0}} .
\end{aligned}
$$

This proposed method is realized to measure the dynamic current stiffness and the dynamic displacement stiffness through adjusting the reference value of the displacement sensor by experiments. The process includes two stages.

The first stage is to determine the magnetic center.

(1) The rotor is suspended stably perpendicular to the direction of gravity with $0 \mathrm{~Hz}$,

(2) The suspended position of rotor is adjusted until the measured current values in the two channels, $z+$ and $z-$ channel, are the same. Thus, the position of the magnetic center is determined.

The second stage is to obtain the dynamic current stiffness and the dynamic displacement stiffness.

(1) Adjust the $z$ channel along the direction of gravity and make the rotor suspend at a specific revolving speed. A sinusoidal signal was injected into the currents of the bearing to excite the closed-loop system.

(2) The corresponding control current $i_{0}(\omega)$ of the magnetic bearing rotor at the magnetic center position is recorded.

(3) The rotor is adjusted to a position $h_{1}$ near the magnetic center, and the corresponding control current is recorded as $i_{1}(\omega)$.

(4) The current stiffness $k_{i}(\omega)$ and the displacement stiffness $k_{h}(\omega)$ at a specific revolving speed can be obtained by Eqs. (32) and (33).

The course of experiment can be represented as a flow diagram shown in Figure 8. 


\section{Measurement and Experiment}

In order to verify the feasibility of the proposed experimental method in this paper, the dynamic stiffness test experiment of AMB is completed in a MSMP platform with the rated speed of $500 \mathrm{~Hz}$ shown in Figure 9. The measured AMB prototype is presented in Figure 10.

\subsection{Dynamic Current Stiffness Measurement and Results}

According to the dynamic stiffness measurement method mentioned in the last chapter, the experiment is carried out in the MSMP, and the corresponding course is as follows.

(1) Keep the $z$ axis direction of MSMP parallel to the floor, and the magnetic center is determined according to the mentioned method in the last chapter.

(2) To measure the dynamic current stiffness, the $z$-axis direction of the MSMP is perpendicular to the floor. The combined force of AMB is the gravity of the rotor.

(3) A sinusoidal signal was injected into the currents of the bearing to excite the closed-loop system. The exciting signal, bearing currents, and displacement of the rotor were measured simultaneously, and the open-loop frequency response obtained. The corresponding dynamic current stiffness under each

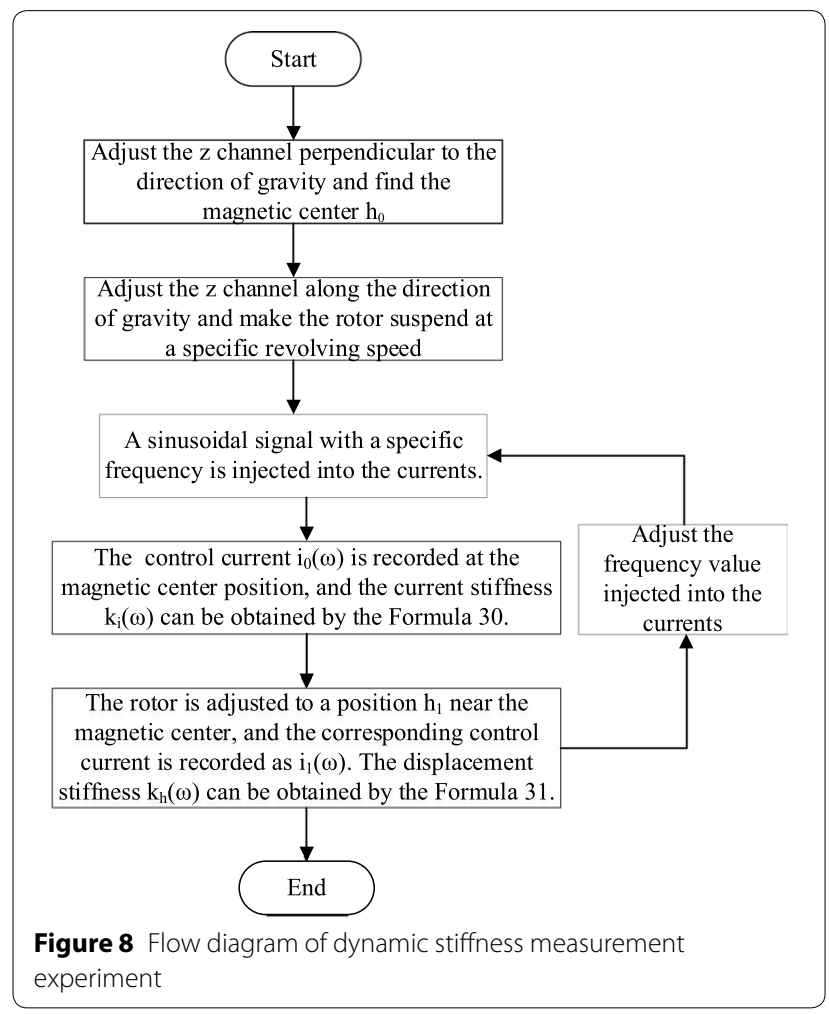

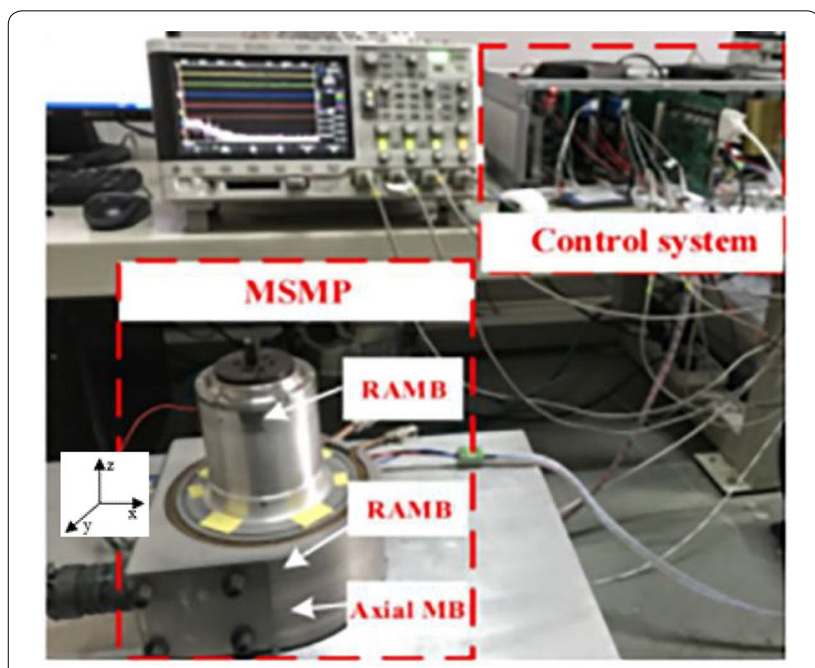

Figure 9 Measurement test system

specific frequency can be obtained through force analysis of the MSMP system and Eq. (32).

The analytical dynamic current stiffness result considering the air gap flux variation compared with the result without considering the air gap flux variation is shown in Figure 11 as the curve 'Analytical Method' (without considering the air gap flux variation) and the curve 'Improved Analytical Method' (considering the air gap flux variation). Compared with the previous analytical method, the dynamic current stiffness result obtained from the improved model decreases by $1.8 \%$. The measured dynamic current stiffness value at the corresponding frequency is shown as the scatter plot in Figure 11. If following the traditional static stiffness measurement

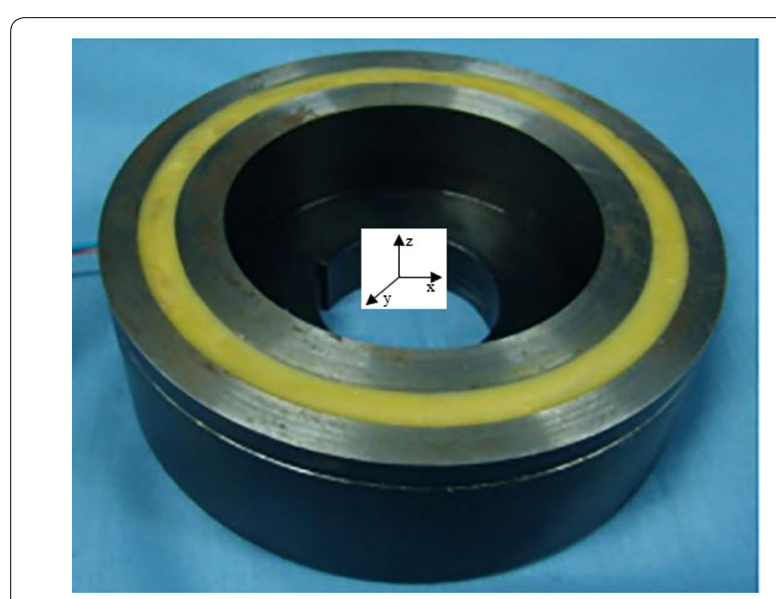

Figure $\mathbf{1 0}$ Prototyped AMB 


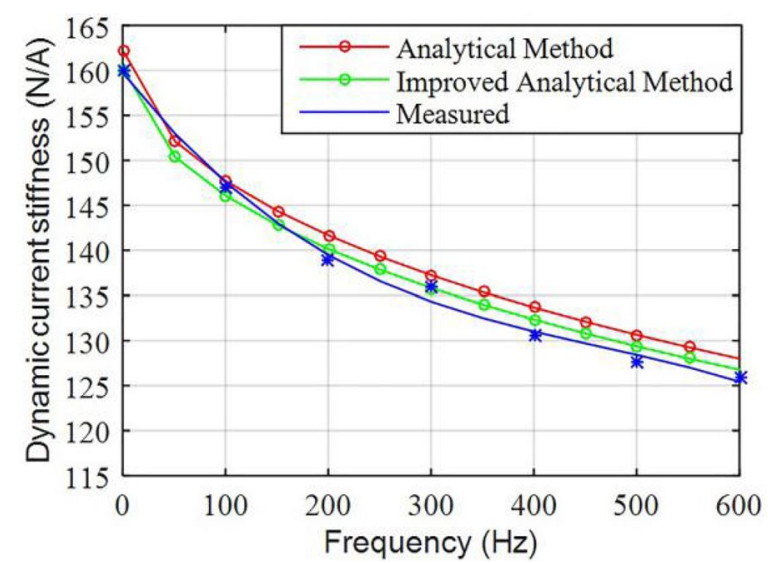

Figure 11 Measured dynamic current stiffness at different frequency compared with analytical results

method, it is obvious the static current stiffness which is under 0 frequency is $160 \mathrm{~N} / \mathrm{A}$, with frequency increasing, from the dynamic stiffness measurement data, we can see the dynamic current stiffness is gradually decreasing to 127.8 N/A, and the error of the dynamic current stiffness compared with the static current stiffness is increasing from $0 \%$ to $20.1 \%$. For measured dynamic current stiffness value, least square regression method was used for dynamic current stiffness-frequency curve fitting with cubic equation. The fitting curve between measured dynamic current stiffness and different frequency compared with analytical results is presented as the curve 'measured' in Figure 11. The maximum error of measured values and analytical values considering the air gap flux variation is $1.3 \%$. The results of experiment performed by the proposed measurement method agreed better with the analytical model considering the air gap flux variation.

\subsection{Dynamic Displacement Stiffness Measurement and Results}

To measure the dynamic displacement stiffness, recording the rotor at a certain non-zero position, the control current at different frequencies can determine the displacement stiffness at the corresponding frequency. In this experiment, the corresponding course is:

(1) The rotor offset from the magnetic center is adjusted to $-0.02 \mathrm{~mm}$.

(2) The dynamic displacement stiffness can be obtained by the recorded control current values under different frequency according to Eq. (33).

The analytical dynamic displacement stiffness result considering the air gap flux variation compared with

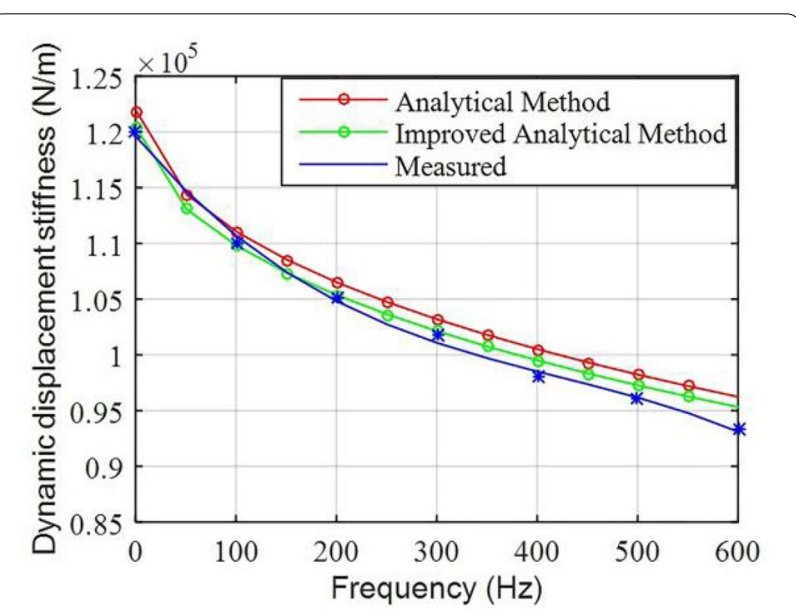

Figure 12 Measured dynamic current stiffness at different frequency compared with analytical results

the result without considering the air gap flux variation is shown in Figure 12 as the curve 'Analytical Method' (without considering the air gap flux variation) and the curve 'Improved Analytical Method' (considering the air gap flux variation). Compared with the previous analytical method, the dynamic displacement stiffness result obtained from the improved model decreases by $1.67 \%$. The measured dynamic displacement stiffness at the corresponding frequency is shown as the scatter plot in Figure 12. According to the static stiffness measurement method, the static displacement stiffness which is under 0 frequency is $1.2 \times 10^{5} \mathrm{~N} / \mathrm{m}$. Using the method of dynamic stiffness measurement method, with frequency increasing, the dynamic displacement stiffness is gradually decreasing to $0.93 \times 10^{5} \mathrm{~N} / \mathrm{m}$, and the error of the dynamic displacement stiffness compared with the static displacement stiffness is increasing from $0 \%$ to $22.5 \%$. For measured dynamic displacement stiffness value, least square regression method was used for dynamic displacement stiffness-frequency curve fitting with cubic equation. The fitting curve between measured dynamic displacement stiffness and different frequency compared with analytical results is presented as the curve 'measured' in Figure 12. The experimental results obtained by the proposed measurement method is consistent with the analytical model with maximum error $2.1 \%$.

It can be seen that there is a certain error between the theoretical dynamic stiffness and the experimental results. The source of the error mainly includes the following aspects: 
(1) Dynamic stiffness testing is performed in the MSMP, and there is inevitably interference from the motor. During the measurement, the electromagnetic field generated by the motor will be superimposed on the electromagnetic field of the magnetic bearing. With the revolving speed increasing, the electromagnetic field generated by the motor changes due to the eddy-current effect. It means that the interference of the motor to the magnetic bearing is very complicated, and it is difficult to eliminate the interference by analyzing.

(2) In the theoretical analysis, some idealized assumptions lead to the error, such as assuming that the material is linear, ignoring magnetic flux leakage, magnetic saturation, and hysteresis.

(3) Through the dynamic stiffness measurement method, the electromagnetic force of the magnetic bearing is a dynamic force related to frequency. The gravity is used to equivalent dynamic electromagnetic force approximately, which leads to the measured force to be less than the actual dynamic force. As a result, the measured dynamic current stiffness at high frequency is smaller than the analytical results.

\section{Conclusions}

This paper firstly builds up the dynamic stiffness model of axial magnetic bearing including the eddy-current effect considering the variation of flux density across the air gap, and then a dynamic stiffness measurement method for AMB in MSMP system is adopted to verify the theoretical analyze results. It is clearly the dynamic stiffness result obtained from the improved model is closer to the experimental fitting curve than the previous analytical result without considering the air gap flux variation. Compared with the static stiffness obtained from the traditional static stiffness measurement method, the error of dynamic current stiffness and displacement stiffness obtained from dynamic stiffness measurement method are from $0 \%$ to $20.1 \%$ and from $0 \%$ to $22.5 \%$, respectively with frequency increasing. The validity of the measurement method has been verified by the experiment performed on the prototyped MSMP. Comparing the experimental value to the theoretical value considering the air gap flux variation, the maximum error of the current stiffness is $1.3 \%$, the maximum error of the displacement stiffness is $2.1 \%$.

The research in this paper has a great progress in the research of the dynamic properties of AMB and provided the evaluation standard for dynamic performance improvement and structure optimization of AMB.

\section{Acknowledgements \\ Not applicable.}

\section{Authors' contributions}

SJ was in charge of the whole analyses. WW wrote the manuscript. TJ and WC assisted with sampling and laboratory analyses. All authors read and approved the final manuscript.

\begin{abstract}
Authors' Information
Jinji Sun, born in 1979, is currently a professor at Beihang University, China. He received his PhD degree from Beihang University, China, in 2010. His research interests include electromagnetic design and control of magnetic bearings, magnetic bearing test and electromagnetic design and control of the motor.
\end{abstract}

Wanting Wei, born in 1993, is currently a master candidate at Key Laboratory of Fundamental Science for National Defense, Novel Inertial Instrument and Navigation System Technology, School of Instrument Science and Opto-electronics Engineering, Beihang University, China.

Jiqiang Tang, born in 1972, is currently a professor at Beihang University, China. He received his PhD degree from Harbin Engineering University, China. His research interests include new magnetic suspension inertial actuator for aerospace and its application technology.

Chun-E Wang, born in 1984, is currently a senior engineer at Beijing Institute of Automatic Control Equipment, China. She received her PhD degree from Beihang University, China.

Funding

Supported by Excellent Youth Science Foundation of China (Grant No. 51722501), National Natural Science Foundation of China (Grant No. 51575025), National Natural Science Foundation of China-Youth Science Foundation (Grant No. 61603052), and Opening Foundation of State Key Laboratory of Advanced Welding and Joining (Grant No. AWJ-20-R02).

\section{Competing interests}

The authors declare no competing financial interests.

\section{Author Details}

${ }^{1}$ Key Laboratory of Fundamental Science for National Defense, Novel Inertial Instrument and Navigation System Technology, School of Instrument Science and Opto-Electronics Engineering, Beihang University, Beijing 100191, China. 2 State Key Laboratory of Advanced Welding and Joining, Harbin Institute of Technology, Harbin 150001, China. ${ }^{3}$ Beijing Institute of Automatic Control Equipment, Beijing 100074, China.

Received: 15 May 2019 Revised: 13 April 2020 Accepted: 15 July 2020 Published online: 29 July 2020

\section{References}

[1] ZY Huang, B C Han, Y Le. Modeling method of the modal analysis for turbomolecular pump rotor blades. Vacuum, 2017, 144: 145-151.

[2] K Mao, G Liu. An improved braking control method for the magnetically levitated TMP with a fast transient response. Vacuum, 2018, 148: 312-318.

[3] Y S Sun. Molecular pump patent preliminary analysis and summary of several key technologies. Modern Manufacturing Technology \& Equipment, 2017. (in Chinese)

[4] Z Z Su, D Wang, J Q Chen, et al. Improving operational performance of magnetically suspended flywheel with PM-biased magnetic bearings using adaptive resonant controller and nonlinear compensation method. IEEE Transactions on Magnetics, 2016, 52(7): 1-4.

[5] B Han, Q Xu, Q Yuan. Multiobjective optimization of a combined radialaxial magnetic bearing for magnetically suspended compressor. IEEE Transactions on Industrial Electronics, 2016, 63(4): 2284-2293.

[6] JT Ju, X B Liu, Z G Xu, et al. Comparison of three-pole AMB and HMB for magnetic suspended molecular pump. Modern Physics Letters B, 2018, 32(5): 18400742.

[7] G Liu, K Mao. Investigation of the rotor temperature of a turbo-molecular pump with different motor drive methods. Vacuum, 2017, 146: 252-258. 
[8] B C Han, ZY Huang, Y Le. Design aspects of a large scale turbomolecular pump with active magnetic bearings. Vacuum, 2017, 142: 96-105.

[9] ZY Huang, B C Han, Y Le. Multidisciplinary design strategies for turbomolecular pumps with ultrahigh vacuum performance. IEEE Transactions on Industrial Electronics, 2019: 1-1.

[10] ZY Yin, Y W Cai, W J Wang, et al. Analysis and experiment of eddy current loss in radial magnetic bearings with solid rotor. Matec Web of Conferences, 2018, 198(6): 04002.

[11] Y Le, J J Sun, B C Han. Modeling and design of 3-DOF magnetic bearing for high-speed motor including eddy-current effects and leakage effects. IEEE Transactions on Industrial Electronics, 2016, 63(6): 3656-3665.

[12] J J Sun, H Zhou, ZY Ju. Dynamic stiffness analysis and measurement of radial active magnetic bearing in magnetically suspended molecular pump. Scientific Reports, 2020, 10(1).

[13] L Kucera, M Ahrens. A model for axial magnetic bearings including eddy currents. The 3rd Intl. Symposium on Magnetic Suspension Technology, 1996.

[14] L Zhu, C R Knospe, E H Maslen. Analytic model for a nonlaminated cylindrical magnetic actuator including eddy currents. IEEE Transactions on Magnetics, 2005, 41(4): 1248-1258.

[15] L Zhu, C R Knospe. Modeling of nonlaminated electromagnetic suspension systems. IEEE/ASME Transactions on Mechatronics, 2010, 15(1): 59-69.

[16] R Seifert, K Rbenack, W Hofmann. Rational approximation of the analytical model of nonlaminated cylindrical magnetic actuators for flux estimation and control. IEEE Transactions on Magnetics, 2019, 55(12): 1-16.

[17] Y Sun, Y S Ho, L Yu. Dynamic stiffnesses of active magnetic thrust bearing including eddy-current effects. IEEE Transactions on Magnetics, 2009, 45(1): 139-149.

[18] X F Hu, G Liu, J J Sun, et al. Analysis on eddy current loss for axial magnetic bearings. Bearing, 2013(03): 22-27.

[19] Y Le, K Wang. Design and optimization method of magnetic bearing for high-speed motor considering eddy current effects. Mechatronics, IEEE/ ASME Transactions on, 2016, 21(4): 2061-2072.
[20] ZW Whitlow, R L Fittro, C R Knospe. Dynamic performance of segmented active magnetic thrust bearings. IEEE Transactions on Magnetics, 2016, 52(11): 1-11.

[21] C H H M Custers, JW Jansen, M C Van Beurden, et al. 3D harmonic modeling of eddy currents in segmented conducting structures. Compel., 2019, 38(1): 2-23.

[22] L Zhou, L Li. Modeling and identification of a solid-core active magnetic bearing including eddy currents. IEEE/ASME Transactions on Mechatronics, 2016, 21(6): 2784-2792.

[23] J J Sun, C E Wang, Y Le. Research on a novel high stiffness axial passive magnetic bearing for DGMSCMG. Journal of Magnetism and Magnetic Materials, 2016, 412(Aug.): 147-155.

[24] J Sun, D Chen, Y Ren. Stiffness measurement method of repulsive passive magnetic bearing in SGMSCMG. IEEE Transactions on Instrumentation and Measurement, 2013, 62(11): 2960-2965.

[25] J J Sun, G C Bai, L Yang. Stiffness measurement of permanent magnet biased radial magnetic bearing in MSFW. Journal of Dynamic Systems, Measurement, and Control, 2015, 137(9): 94505.

[26] J J Sun, G C Bai, L J Li. Stiffness measurement of radial hybrid magnetic bearing in MSFW. Transactions of the Institute of Measurement and Control, 2015, 37(8): 991-998.

[27] R Z Zhu, W Xu, CY Ye, et al. Novel heteropolar radial hybrid magnetic bearing with low rotor core loss. IEEE Transactions on Magnetics, 2017, (99): 1.

[28] Y Le, J C Fang, B C Han, et al. Dynamic circuit model of a radial magnetic bearing with permanent magnet bias and laminated cores. International Journal of Applied Electromagnetics and Mechanics, 2014, 46(1): 43-60.

[29] J J Sun, H Zhou, X Ma, et al. Study on PID tuning strategy based on dynamic stiffness for radial active magnetic bearing. ISA Transactions, 2018, 2018: S001905781830291X-.

\section{Submit your manuscript to a SpringerOpen ${ }^{\odot}$ journal and benefit from:}

- Convenient online submission

- Rigorous peer review

- Open access: articles freely available online

- High visibility within the field

- Retaining the copyright to your article

Submit your next manuscript at $\boldsymbol{\nabla}$ springeropen.com 